CARDIOVASCULAR MEDICINE

\title{
Increased risk of heart failure as a consequence of perioperative myocardial injury after coronary artery bypass grafting
}

\author{
J Stever, F Granath, U de Faire, A Ekbom, E Ståhle
}

Heart 2005;91:754-758. doi: 10.1136/hrt.2004.035048

See end of article for authors' affiliations

Correspondence to: Johnny Stever, MD, Department of Cardiothoracic Surgery, University Hospital, SE75185 Uppsala, Sweden; johnny.steuer@surgsci.uu.

Accepted 28 July 2004
Objective: To analyse the relation between perioperative myocardial injury (PMI) and the risk of subsequent heart failure after coronary artery bypass grafting (CABG).

Design and setting: Clinical data were documented prospectively in all patients and stored in a computer. All hospital readmissions were identified and the registered primary diagnoses were analysed. Survival information on all patients was obtained by use of combined registers. The study was carried out at the cardiac surgical referral centre of University Hospital, Uppsala, Sweden.

Patients: 7493 patients discharged alive after primary CABG between 1987 and 1996 were followed up until the first hospital readmission for heart failure, death, or 31 December 1996 was reached.

Main outcome measures: Hospital readmission for heart failure or late mortality.

Results: Of the patients studied 576 (7.7\%) were readmitted for heart failure. Actuarial freedom from readmission for heart failure after four years was $93 \%$, and after seven years, $89 \%$. Of the 576 patients, $114(20 \%)$ had had PMI, which increased the risk of heart failure independently (hazard ratio (HR) 2.3, $95 \%$ confidence interval (Cl) 1.8 to 2.8). Increased age, female sex, diabetes, previous myocardial infarction, dyspnoea, preoperative atrial fibrillation, left ventricular dysfunction, and triple vessel disease were independent risk factors for heart failure. The use of an internal mammary artery decreased the risk. PMI implied increased mortality (HR $1.4,95 \% \mathrm{Cl} 1.1$ to 1.8). Late mortality was greatly increased in patients readmitted for heart failure.

Conclusion: PMI increased the risk of heart failure and late death after CABG, and heart failure had a notable adverse effect on late survival.
M yocardial infarction, or irreversible myocardial injury, is an important cause of hospital complications and death after cardiac surgery. ${ }^{1-3}$ The long term clinical consequences have not been thoroughly studied and are therefore still unclear. Perioperative myocardial infarction, defined as a new Q wave on the postoperative ECG, has been shown to increase the risk of subsequent congestive heart failure. ${ }^{3}$ There is growing evidence that elevated concentrations of serum cardiac markers after coronary artery bypass grafting (CABG) infer adverse long term survival ${ }^{4}$ as well as increased morbidity in the long term. ${ }^{5}$

Measurement of serum concentrations of biochemical markers has long been used to detect myocardial damage following acute myocardial infarction or cardiac surgery. Aspartate aminotransferase (ASAT) was the first marker to be introduced in clinical routine. ${ }^{6}$ This was followed by the introduction of other, more specific diagnostic markers, such as creatine kinase myocardial band (CK-MB), troponin $\mathrm{T}$, and troponin I. ${ }^{6}$

Perioperative myocardial injury (PMI) may be the consequence of early graft closure, ineffective myocardial protection, or both. ${ }^{8}$ Irrespective of the underlying cause, it can be hypothesised that PMI will lead to impaired left ventricular function and consequently heart failure.

The aims of this study were to analyse the long term occurrence of heart failure after CABG, to identify risk factors, and in particular to elucidate the relation between PMI and the risk of subsequent heart failure. A further aim was to assess the effects of PMI and heart failure on long term survival.

\section{METHODS}

\section{Patients}

From 1 January 1987 to 31 December 1996, 7721 adult patients underwent primary, isolated CABG at the department of cardiothoracic surgery of the University Hospital, Uppsala, Sweden. Among these, 228 patients (3.0\%) died during the initial period of hospitalisation, leaving 7493 patients discharged alive; these patients constituted the basis of the present study (table 1 ).

\section{Data collection}

Serum ASAT and serum alanine aminotransferase (ALAT) activities were measured before surgery and on the first postoperative morning (day 1). The methods of quantifying serum ASAT activity were fairly consistent throughout the study period. Up to 1989 the instrument used was the Greiner 300, and after that time the Hitachi 717 was used. Serum CKMB was also measured, but up to 1992 several methods were used to evaluate the enzymatic activity. In the period 1993-96 one sole method was used to quantify CK-MB mass (Abbott IMx).

Clinical data were documented prospectively, according to a protocol, in all patients and stored in a computer. ${ }^{4}$ Hypertension was defined as antihypertensive medication or blood pressure $\geqslant 140 / 90 \mathrm{~mm} \mathrm{Hg}$ on admission; diabetes

\footnotetext{
Abbreviations: ASAT, aspartate aminotransferase; ALAT, alanine aminotransferase; $C A B G$, coronary artery bypass grafting; $C K-M B$, creatine kinase myocardial band; $E F$, ejection fraction; $H R$, hazard ratio; ICD, International classification of diseases; NYHA, New York Heart Association; PMI, perioperative myocardial injury
} 
Table 1 Patient and surgical variables and hazard ratios (HR) for readmission because of heart failure from a multivariate model with $95 \%$ confidence intervals $(\mathrm{CI})$.

\begin{tabular}{|c|c|c|c|c|c|c|}
\hline & \multicolumn{2}{|c|}{$\begin{array}{l}\text { All patients } \\
\text { ( } n=7493 \text { ) }\end{array}$} & \multicolumn{4}{|c|}{ Patients readmitted for heart failure $(n=576)$} \\
\hline & $\mathbf{n}$ & $\%$ & $\mathbf{n}$ & $\%$ of all & HR & $95 \% \mathrm{Cl}$ \\
\hline \multicolumn{7}{|l|}{ Sex } \\
\hline Male & 5905 & 79 & 434 & 7 & Reference & \\
\hline Female & 1588 & 21 & 142 & 9 & $1.3^{*}$ & 1.1 to 1.6 \\
\hline \multicolumn{7}{|c|}{ Age at time of surgery (years) } \\
\hline$\leqslant 49$ & 690 & 9 & 34 & 5 & Reference & \\
\hline $50-59$ & 1947 & 26 & 95 & 5 & $0.7^{*}$ & 0.5 to 0.9 \\
\hline $60-69$ & 3284 & 44 & 294 & 9 & 1.2 & 0.9 to 1.5 \\
\hline$\geqslant 70$ & 1572 & 21 & 153 & 10 & $1.4^{*}$ & 1.1 to 1.9 \\
\hline \multicolumn{7}{|l|}{ Smoking status } \\
\hline Never smoked & 2564 & 35 & 182 & 7 & Reference & \\
\hline Ex-smoker & 4102 & 55 & 340 & 8 & 1.2 & 1.0 to 1.5 \\
\hline Current smoker & 781 & 10 & 54 & 7 & 1.4 & 1.0 to 1.9 \\
\hline \multicolumn{7}{|l|}{ Hypertension } \\
\hline No & 4752 & 63 & 358 & 8 & Reference & \\
\hline Yes & 2741 & 37 & 218 & 8 & 1.1 & 0.9 to 1.3 \\
\hline \multicolumn{7}{|l|}{ Diabetes mellitus } \\
\hline No & 6383 & 85 & 453 & 7 & Reference & \\
\hline Yes & 1110 & 15 & 123 & 11 & $1.5^{\star}$ & 1.2 to 1.8 \\
\hline \multicolumn{7}{|l|}{ Previous Mls } \\
\hline None & 2898 & 39 & 114 & 4 & Reference & \\
\hline 1 & 3109 & 41 & 229 & 7 & $1.4^{*}$ & 1.1 to 1.8 \\
\hline$\geqslant 2$ & 1482 & 20 & 233 & 16 & $2.3^{*}$ & 1.8 to 3.0 \\
\hline \multicolumn{7}{|l|}{ Angina status } \\
\hline Stable & 6038 & 81 & 426 & 7 & Reference & \\
\hline Unstable & 1454 & 19 & 149 & 10 & 1.2 & 1.0 to 1.6 \\
\hline \multicolumn{7}{|l|}{ Dyspnoea } \\
\hline No & 5744 & 77 & 390 & 7 & Reference & \\
\hline Yes & 1749 & 23 & 186 & 11 & $1.6^{*}$ & 1.4 to 2.0 \\
\hline \multicolumn{7}{|c|}{ Preoperative atrial fibrillation } \\
\hline No & 5534 & 74 & 406 & 7 & Reference & \\
\hline Yes & 1959 & 26 & 170 & 9 & $1.3^{*}$ & 1.1 to 1.6 \\
\hline \multicolumn{7}{|l|}{ Left ventricular function } \\
\hline Intact & 4530 & 61 & 196 & 4 & Reference & \\
\hline Moderate dysfunction & 2486 & 33 & 266 & 11 & $1.8^{*}$ & 1.5 to 2.2 \\
\hline Severe dysfunction & 474 & 6 & 114 & 24 & $3.8^{*}$ & 2.9 to 4.8 \\
\hline \multicolumn{7}{|l|}{ NYHA class } \\
\hline$|-| \mid$ & 352 & 5 & 12 & 3 & Reference & \\
\hline IIIA & 2959 & 39 & 154 & 5 & 1.1 & 0.6 to 2.0 \\
\hline IIIB & 3488 & 47 & 335 & 10 & 1.4 & 0.8 to 2.5 \\
\hline IV & 693 & 9 & 75 & 11 & 1.4 & 0.7 to 2.8 \\
\hline \multicolumn{7}{|l|}{ Coronary stenoses } \\
\hline 1 & 472 & 6 & 18 & 4 & Reference & \\
\hline 2 & 1695 & 23 & 100 & 6 & 1.5 & 0.9 to 2.5 \\
\hline 3 & 5318 & 71 & 458 & 9 & $1.8^{*}$ & 1.1 to 3.0 \\
\hline \multicolumn{7}{|l|}{ Left main stem stenosis } \\
\hline No & 5869 & 78 & 438 & 7 & Reference & \\
\hline Yes & 1624 & 22 & 138 & 9 & 1.1 & 0.9 to 1.3 \\
\hline \multicolumn{7}{|l|}{$\mathrm{CP}$ bypass time ( $\mathrm{min}$ ) } \\
\hline$\leqslant 119$ & 6356 & 85 & 430 & 7 & Reference & \\
\hline \multirow{2}{*}{\multicolumn{7}{|c|}{$\geqslant 120$}} \\
\hline & & & & & & \\
\hline No & 897 & 12 & 107 & 12 & Reference & \\
\hline Yes & 6596 & 88 & 469 & 7 & $0.8^{*}$ & 0.6 to 0.9 \\
\hline PMl† & & & & & & \\
\hline No & 6802 & 91 & 462 & 7 & Reference & \\
\hline Yes & 691 & 9 & 114 & 16 & $2.3^{*}$ & 1.8 to 2.8 \\
\hline
\end{tabular}

mellitus was defined as treatment with insulin or drugs. Classification of congestive heart failure according to the New York Heart Association (NYHA) system was based on a pre-operative interview with the patient. A subdivision of patients in NYHA class III into IIIA and IIIB was applied. ${ }^{4}$ Left ventricular function was categorised as: intact, ejection fraction $(\mathrm{EF}) \geqslant 0.50$; moderate dysfunction, EF $0.30-0.49$; or severe dysfunction, EF $<0.30$.

\section{Follow up and outcome}

A unique 10 digit national registration number is allocated to every Swedish resident. In January 1997 survival information on all patients was obtained by computerised linkage to two national registers, namely a continuously updated census register and the Swedish Cause of Death Register. By use of these combined registers, all patients could be identified as being alive on 31 December 1996 or assigned a date of death.

Heart failure was defined as hospital readmission for heart failure. Cause of readmission was recorded on the basis of the International classification of diseases, 9th revision (ICD-9), and was categorised by means of the primary discharge diagnosis. Heart failure was recorded as the ICD-9 code 428. The date of readmission is designated the date of event. All hospital readmissions from the date of surgery until 31 December 
1996 were identified and the registered primary diagnoses at discharge were analysed by computerised linkage with the Swedish Hospital Discharge Register (Centre for Epidemiology, National Board of Health and Welfare). The date of discharge following the admission for CABG (index episode) was used as the starting point.

\section{Statistical methods}

The Kaplan-Meier method was used to calculate life table estimates for readmission for heart failure. ${ }^{9}$

Risk factors for heart failure were analysed using the Cox proportional hazards model. Results are presented as hazard ratio (HR) and 95\% confidence interval (CI). The relation between serum ASAT and risk of heart failure was explored, using serum ASAT concentrations categorised into deciles in univariate analyses. The 10 decile, serum ASAT $\geqslant 2.55 \mu \mathrm{kat} / \mathrm{l}$, was the optimal dichotomised form-that is, the form with the best discriminatory power-and was the basis for the definition of PMI. In addition, an ASAT:ALAT ratio was calculated to distinguish serum ASAT elevation caused by cardiac conditions, from that caused by hepatic congestion or the side effects of drugs. An ASAT increase with a simultaneous ASAT:ALAT ratio of $\geqslant 1.5$ was considered to be of primarily cardiac origin. ${ }^{10}{ }^{11}$ PMI was defined as serum ASAT $\geqslant 2.55 \mu \mathrm{kat} / \mathrm{l}$ and ASAT:ALAT $\geqslant 1.5$. Furthermore, within the 10th decile the incremental risk associated with serum ASAT concentrations corresponding to the 95th and to the 99th centile was investigated.

Interactions between PMI and left ventricular function, previous myocardial infarctions, dyspnoea, hypertension, sex, and use of the internal mammary artery, were sequentially tested with likelihood ratio tests.

The persistence of the effect of having sustained a PMI over time after surgery was investigated by allowing effects to change with two year intervals after surgery. The significance of this effect was tested by introducing an interaction term between the indicator for PMI and time since surgery as a continuous variable.

The influence of readmission for heart failure on subsequent survival was analysed by exploring the age adjusted effect of PMI on mortality before and after the first inhospital episode with heart failure. This was accomplished by a Cox regression model for patients with and without PMI, including a time dependent indicator variable, which switches from 0 to 1,30 days after the first readmission for heart failure.

The concordance between serum ASAT $\geqslant 2.55 \mu \mathrm{kat} / \mathrm{l}$ and serum $\mathrm{CK}-\mathrm{MB} \geqslant 50 \mu \mathrm{g} / \mathrm{l}$, the latter chosen as an analogous cut off for peri-procedural myocardial injury, was analysed for the period 1993-96.

The Statistical Analysis System (SAS) package was used for all statistical analyses.

\section{RESULTS}

Of the 7493 patients, $576(7.7 \%)$ were readmitted for heart failure. Actuarial freedom from readmission for heart failure after one year was $97 \%$ (95\% CI $96 \%$ to $97 \%$ ), after four years, 93\% (95\% CI 93\% to $94 \%$ ) and after seven years, $89 \%$ (95\% CI $88 \%$ to $90 \%)$.

\section{Relation between serum ASAT and heart failure}

The relation between serum ASAT and the risk of heart failure was not linear. The 10th decile (equivalent to $\geqslant 2.55 \mu \mathrm{kat} / \mathrm{l}$ ) was used as cut off since no increase in risk was seen below this concentration. Of the patients readmitted for heart failure, six patients had concentrations above this cut off but had an ASAT:ALAT ratio < 1.5; these patients did not show an elevated risk of heart failure (HR $1.1,95 \%$ CI 0.5 to 2.5 ). Within the highest decile there was a

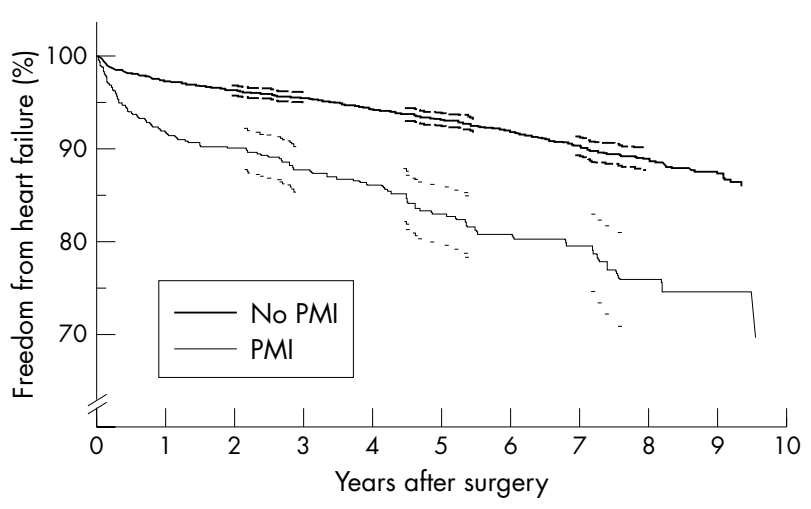

Figure 1 Freedom from readmission for heart failure in relation to perioperative myocardial injury (PMI); $95 \%$ confidence intervals are shown at the time points $2.5,5$ and 7.5 years after surgery.

positive relation between serum ASAT and risk of heart failure: serum ASAT 2.55-3.46 $\mu \mathrm{kat} / \mathrm{l}$ (HR 1.8, 95\% CI 1.3 to 2.4); 3.46-6.80 $\mu \mathrm{kat} / \mathrm{l}$ (HR 2.3, 95\% CI 1.7 to 3.1); and $>6.80 \mu \mathrm{kat} / \mathrm{l}$ (HR 3.6, 95\% CI 2.2 to 5.7 ).

\section{Risk factors for heart failure}

Of the 576 patients who sustained heart failure, $114(20 \%)$ had had a PMI, which increased the risk of heart failure (fig 1) (multivariate HR 2.3, 95\% CI 1.8 to 2.8). As expected, patients with severe left ventricular dysfunction had a high risk of heart failure (HR 3.8, 95\% CI 2.9 to 4.8). Moderate dysfunction also implied an increased risk (HR 1.8, 95\% CI 1.5 to 2.2 ) as compared to preserved left ventricular function. Also, other potential measures of the status of the left ventricle, such as history of a previous myocardial infarction (HR 1.4, 95\% CI 1.1 to 1.8 ) or more than one previous myocardial infarction (HR 2.3, 95\% CI 1.8 to 3.0), dyspnoea (HR 1.6, 95\% CI 1.4 to 2.0), and preoperative atrial fibrillation (HR 1.3, 95\% CI 1.1 to 1.6), were independent risk factors for heart failure. Among the other risk modifiers (table 1), it was found that the use of an internal mammary artery decreased the risk (HR 0.8, 95\% CI 0.6 to 0.9 ). There was no substantial confounding of the effect of elevated serum ASAT by the other risk factors (that is, the univariate estimate was affected less than $10 \%$ after adjustment for all other risk factors, data not presented).

\section{Interaction analyses}

There were no significant interaction effects. However, there was a borderline significant interaction between PMI and number of previous myocardial infarctions $(p=0.06)$, suggesting that the risk associated with elevated serum ASAT was more pronounced in patients with a previous myocardial infarction. In the other situations, the relative effect of PMI was virtually the same in all subgroups-that is, no significant deviations from multiplicity were detected. The effect of PMI in patients with varying left ventricular function illustrates the multiplicative effect. In patients with severe preoperative left ventricular dysfunction and an additional PMI, the hazard ratio of heart failure was more than eight times that in patients with intact left ventricular function and no perioperative injury (fig 2).

\section{Time dependence of PMI}

No tendency was observed towards a decrease over time in the effect of PMI on the risk of heart failure (table 2).

\section{Long term mortality}

PMI implied an increased all cause mortality. PMI and no heart failure, HR 1.4 (95\% CI 1.1 to 1.8 ); patients with no 

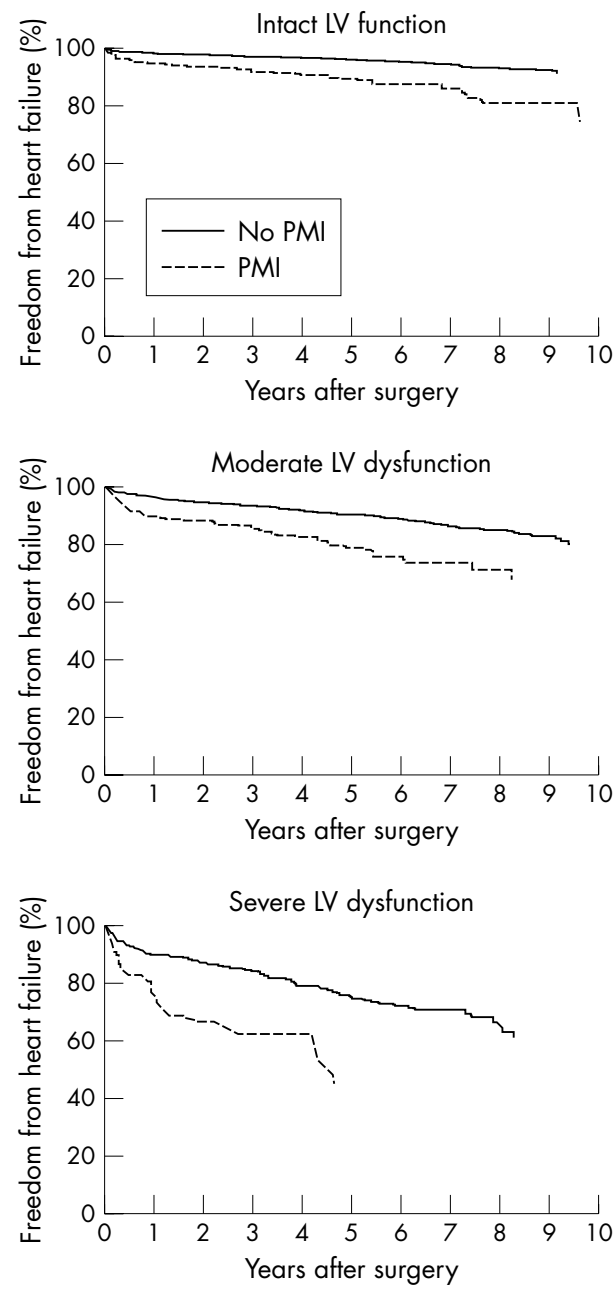

Figure 2 Combined effect of perioperative myocardial injury (PMI) and left ventricular (LV) function on readmission for heart failure.

PMI and no heart failure constituted the reference group with HR 1.0. After readmission for heart failure survival was poor irrespective of whether the patient had sustained a PMI or not (PMI and heart failure, HR 4.5, 95\% CI 3.1 to 6.7; no PMI and heart failure, HR 5.7, 95\% CI 4.6 to 7.0 ), compared to the reference group-that is, patients with no PMI and no heart failure.

\section{Concordance between serum ASAT and serum CK-MB} During 1993-96, 76\% of the patients with serum ASAT $\geqslant 2.55 \mu \mathrm{kat} / \mathrm{l}$ also had serum CK-MB $\geqslant 50 \mu \mathrm{g} / \mathrm{l}$. Among the patients with serum ASAT $<2.55 \mu \mathrm{kat} / \mathrm{l}$ - that is, no PMI93\% had serum CK-MB $<50 \mu \mathrm{g} / \mathrm{l}$.

\section{DISCUSSION}

This study clearly established that PMI increased the risk of heart failure after CABG. The risk associated with such an event was independent of other risk factors and persisted for many years after surgery. PMI was also associated with increased mortality, but all patients readmitted for heart failure had notably decreased long term survival irrespective of prior PMI.

\section{PMI and heart failure after CABG}

Among the patients with PMI, 17\% had developed heart failure within five years of surgery, compared to $7 \%$ of those without PMI. The adverse outcome observed in association
Table 2 Long term effect of perioperative myocardial injury $(\mathrm{PMI})^{*}$ as a risk factor for heart failure

\begin{tabular}{|c|c|}
\hline Time after surgery (years) & $\mathrm{HRt}(95 \% \mathrm{Cl})$ \\
\hline $\begin{array}{l}0-2 \\
2-4 \\
4-6 \\
6-8 \\
>8 \\
\text { Trend test } \neq\end{array}$ & $\begin{array}{l}2.3(1.8 \text { to } 3.0) \\
1.9(1.0 \text { to } 3.4) \\
2.4(1.3 \text { to } 4.7) \\
1.3(0.5 \text { to } 3.6) \\
2.1(0.5 \text { to } 9.6) \\
p=0.31\end{array}$ \\
\hline \multicolumn{2}{|c|}{$\begin{array}{l}\text { *PMI is classified as serum AMAT } \geqslant 2.55 \mu \mathrm{kat} / \mathrm{I} \text { with } \\
\text { AMAT:ALAT ratio } \geqslant 1.5 ; \text { THR (reference }=\text { serum ASAT } \\
<2.55 \mu \text { kat/l) are given as a function of time after surgery } \\
\text { and adjusted for the same variables as in table } 1 \text {; } \\
\text { finteraction with time after surgery as a continuous variable }\end{array}$} \\
\hline
\end{tabular}

with PMI could simply be the effect of necrosis and loss of myocardial cells. Our results argue against the alternative explanation that an increase in cardiac markers is a benign phenomenon, reflecting a reversible injury caused by profound myocardial ischaemia during surgery, but with preserved cellular viability. ${ }^{12}$ It is also conceivable that increased release of cardiac markers reflects a poor surgical result in terms of graft patency. Moreover, the same factors that make the patient susceptible to a myocardial injury may also increase the risk of subsequent heart failure. However, in the present study PMI had a pronounced effect on the risk of subsequent heart failure, with a hazard ratio of 2.3, even after correction for other risk factors. This finding strongly indicates a causative effect.

Previous studies of the long term consequences of PMI have mostly demonstrated a negative effect in the short and intermediate term. In a 30 month follow up study, patients with Q wave perioperative myocardial infarction were found to be more likely to have cardiac events. ${ }^{3}$ In another study, Q wave perioperative infarction implied a three year survival of $85 \%$, compared to $95 \%$ among patients without such an event. ${ }^{13}$ In a recent report, ${ }^{5}$ patients with CK-MB concentrations more than five times normal were at increased risk of death and myocardial infarction at one year of follow up. In a previous study, we found that after CABG, ASAT and CK-MB provided equal prognostic information about the risk of both early and late death. The long term deleterious consequences of elevation of either one or both of these markers on survival persisted for many years after surgery. ${ }^{4}$ Hence, the decreased survival associated with increased cardiac markers can at least partly be explained by an increased incidence of heart failure.

Our definition of heart failure was based on data from the Swedish Hospital Discharge Register, which covers all public, inpatient care in Sweden. In a validation study, examination of a national sample of patients with ischaemic heart disease indicated a positive predictive value of $86 \%$ for ICD- 9 code 410 (acute myocardial infarction) in discharge data. ${ }^{14}$ Diagnostic accuracy rates of the same magnitude have been demonstrated in additional studies, ${ }^{15}{ }^{16}$ suggesting that there may be an underestimation of the occurrence of heart failure. ${ }^{15}$

\section{Other risk factors for heart failure after CABG}

As expected, left ventricular function had a high impact on the risk of heart failure, with an almost fourfold increase in patients with severe dysfunction. We hypothesised that PMI would be more dangerous in patients with impaired left ventricular function, but such an interaction was not found. PMI resulted in the same relative risk addition within each category of left ventricular function and other risk factors. However, the multiplicative effect infers a larger increase in 
the absolute risk of heart failure. For example, patients with intact left ventricular function had a five year incidence of heart failure of $4 \%$ in the absence of PMI and $10 \%$ if they had sustained a PMI. Corresponding figures for patients with severe left ventricular dysfunction were $25 \%$ and 55\%, respectively.

NYHA functional class, which should at least partly reflect the left ventricular function under stress, had a less pronounced effect. Furthermore, the number of previous myocardial infarctions was used as a surrogate measure of myocardial scarring in order to differentiate between myocardial fibrosis and hibernation. Patients with at least two previous myocardial infarctions had a more than twofold risk of subsequent heart failure after correction for other factors.

Compared with men, women exhibited a $30 \%$ higher risk of heart failure. Women have been shown to have a higher incidence of in-hospital congestive heart failure or pulmonary oedema after $\mathrm{CABG},{ }^{17}$ and in patients undergoing coronary angiography female sex is an independent predictor of congestive heart failure. ${ }^{18}$

\section{Definition of PMI}

In clinical practice today, CK-MB, troponin $\mathrm{T}$, and troponin I are more widely used than ASAT and considered more specific and sensitive as indicators of myocardial damage. ${ }^{2}$ Nevertheless, serum ASAT values were available during the entire study period and for all patients, and after correction for concomitant serum ALAT elevation, serum ASAT should provide a fairly reliable measure of myocardial injury. We find it reasonable to conclude that increased release of cardiac markers, reflecting myocardial injury or infarction, implies an increase in the risk of subsequent heart failure. The presence of new Q waves on the postoperative ECG is another commonly used definition of perioperative myocardial infarction. The diagnostic reliability of this finding has been questioned, however, and follow up studies of patients with new $\mathrm{Q}$ waves after CABG have yielded conflicting results. ${ }^{3} 19$

In summary, PMI is a serious adverse event, contributing to a clearly increased risk of subsequent heart failure, and has an adverse effect on long term survival. The risk of heart failure persisted for many years after surgery. Heart failure, irrespective of PMI, notably increased late mortality. The clinical implication of our findings is that patients with cardiac enzyme concentrations suggestive of myocardial injury should be regarded as a high risk group for adverse events after discharge and should thus be followed up and treated accordingly.

\section{ACKNOWLEDGEMENTS}

Financial support was provided by the Swedish Heart Lung Foundation.

\section{Authors' affiliations}

J Stever, E Ståhle, Department of Cardiothoracic Surgery, University Hospital, Uppsala, Sweden

F Granath, A Ekbom, Unit of Clinical Epidemiology, Department of Medicine, Karolinska Hospital, Karolinska Institute, Stockholm, Sweden U de Faire*, Division of Cardiovascular Epidemiology, National Institute of Environmental Medicine, Karolinska Institute

*Also at Cardiovascular Laboratory, Department of Cardiology, Karolinska Hospital

\section{REFERENCES}

1 Galinanes $M$. In search of a reliable marker of tissue injury during heart surgery [editorial]. Heart 1998;80:317-8.

2 Holmvang L, Jurlander B, Rasmussen C, et al. Use of biochemical markers of infarction for diagnosing perioperative myocardial infarction and early graft occlusion after coronary artery bypass surgery. Chest 2002;121:103-11.

3 Force T, Hibberd P, Weeks G, et al. Perioperative myocardial infarction after coronary artery bypass surgery. Clinical significance and approach to risk stratification. Circulation 1990;82:903-12.

4 Stever J, Hörte LG, Lindahl B, et al. Impact of perioperative myocardial injury on early and long-term outcome after coronary artery bypass grafting. Eur Heart $J$ 2002;23:1219-27.

5 Costa MA, Carere RG, Lichtenstein SV, et al. Incidence, predictors, and significance of abnormal cardiac enzyme rise in patients treated with bypass surgery in the arterial revascularization therapies study (ARTS). Circulation 2001; 104:2689-93

6 Adams JE III, Abendschein DR, Jaffe AS. Biochemical markers of myocardial injury. Is MB creatine kinase the choice for the 1990s? Circulation 1993;88:750-63.

7 Carrier M, Pellerin M, Perrault LP, et al. Troponin levels in patients with myocardial infarction after coronary artery bypass grafting. Ann Thorac Surg 2000;69:435-40.

8 Jain U. Myocardial infarction during coronary artery bypass surgery. J Cardiothorac Vasc Anesth 1992;6:612-23.

9 Kaplan EL, Meier P. Nonparametric estimation from incomplete observations. $J$ Am Stat Assn 1958;53:457-81.

10 Wallerstedt S, Olsson R, Waldenström J. The diagnostic significance of a high ASAT-ALAT (GOT-GPT) ratio in patients with very high serum aminotransferase levels. Acta Med Scand 1974;195:227-9.

11 Rej R. Aminotransferases in disease [review]. Clin Lab Med 1989;9:667-87.

12 Califf RM, Abdelmeguid AE, Kuntz RE, et al. Myonecrosis after revascularization procedures. J Am Coll Cardiol 1998;31:241-51.

13 Chaitman BR, Alderman EL, Sheffield LT, et al. Use of survival analysis to determine the clinical significance of new $Q$ waves after coronary bypass surgery. Circulation 1983;67:302-7.

14 Hammar N, Alfredsson L, Rosén M, et al. A national record linkage to study acute myocardial infarction incidence and case fatality in Sweden. Int J Epidemiol 2001;30:S30-4.

15 Goff DC Jr, Pandey DK, Chan FA, et al. Congestive heart failure in the United States: is there more than meets the I(CD code)? The Corpus Christi heart project. Arch Intern Med 2000; 160:197-202.

16 Campbell SE, Campbell MK, Grimshaw JM, et al. A systematic review of discharge coding accuracy. J Public Health Med 2001;23:205-11.

17 Jacobs AK, Kelsey SF, Brooks MM, et al. Better outcome for women compared with men undergoing coronary revascularization: a report from the bypass angioplasty revascularization investigation (BARI). Circulation 1998;98:1279-85.

18 Mendes LA, Davidoff R, Cupples LA, et al. Congestive heart failure in patients with coronary artery disease: the gender paradox. Am Heart $J$ 1997;134:207-12.

19 Brasch AV, Khan SS, Denton TA, et al. Twenty-year follow-up of patients with new perioperative $Q$ waves after coronary artery bypass grafting. Am J Cardiol 2000;86:677-9. 